\title{
The NMSSM implementation in WHIZARD
}

\author{
Jürgen Reuter and Felix Braam \\ University of Freiburg, Institute of Physics, Hermann-Herder-Str. 3, 79104 Freiburg, Germany
}

\begin{abstract}
The Next-To-Minimal-Supersymmetric extension of the Standard Model (NMSSM) has been in the focus of extensive studies in the past two decades. In anticipation of the LHC era, the interest in automatized tools that can calculate collider signatures has grown. We present the implementation of the NMSSM into the event generator WHIZARD. In addition to a brief review of the implementation, we discuss the testing and validation procedure. Phenomenological studies will not be presented here.
\end{abstract}

Keywords: NMSSM, Extended supersymmetric models, Monte Carlo Event Generators PACS: $11.30 . \mathrm{Pb}, 11.80 . \mathrm{Cr}, 12.60 . \mathrm{Jv}, 14.80 . \mathrm{Ly}$

\section{THE MULTI-PURPOSE EVENT GENERATOR WHIZARD}

WHIZARD [1] is a multi-purpose Monte-Carlo event generator for the Standard Model (SM) and beyond (BSM). It uses a multi-channel adaptive phase space integration based on the VAMP algorithm [2], which is called by a highly efficient phase space grid decomposition relying on heuristics of the underlying resonance structure of field theoretic scattering amplitudes. The matrix element are delivered by the generator O'Mega [3] which avoids in an optimal way all redundancies of tree-level scattering amplitudes and combines gauge-invariant substructures. WHIZARD was the first generator for full matrix elements for the MSSM [4] and has been validated by many groups and projects both for supersymetric models and alternative BSM models [5]. Among the available models are half a dozen variants of Little Higgs models, extra-dimensional models, several (extended) supersymmetric models, general anomalous gauge, top and Higgs couplings, general unitarization models for electroweak scattering processes, noncommutative versions of the Standard Model etc.

WHIZARD can be accessed via the Hepforge web site: http://projects . hepforge.org/whiza The alpha version of release 2.0.0 has been finished just before the SUSY conference, including the first implementation of the Next-to-Minimal Standard Model (NMSSM) in a multi-particle event generator, which is the main topic here. We briefly want to review the new features of version 2.0.0, which brings a major improvement over the older version 1 . WHIZARD has been completely restructured in a fully object-oriented way. It allows for an arbitrary setup of structure functions, comprises a fully flexible script language for defining scales, cuts, analyses variables as arbitrary functions of kinematic variables and particle lists, it contains a rudimentary own parton shower (which will be further developed during the development of WHIZARD 2 and connected with a module for simulating multiple interactions which is right now being developed). WHIZARD is able to write out standard event formats like LHA, LHEF, STDHEP, HepMC etc. It comes with its own graphics analysis tool, while an interface to ROOT 
will also be available in WHIZARD 2. Also, an interface to DELPHES [6] for a fast detector simulation is under way.

Although, WHIZARD is able to deliver matrix elements for $2 \rightarrow 16$ processes (or higher multiplicities) and integrate processes with at least up to ten particles in the final states, this might not be sufficient for BSM models with a discrete parity symmetry as suggested by the existence of dark matter. Hence, WHIZARD 2 is also able to integrate and simulate long cascade decay chains with full spin correlations. A further improvement is a full-fledged interface to FeynRules [7] for a more automatized inclusion of new physics models for both WHIZARD versions 1 and 2.

\section{THE NMSSM}

The Next-to-Minimal Supersymmetric Standard Model (NMSSM) is a viable extension of the MSSM in the Higgs sector of the model (see e.g. the references in [8]). The main motivation for the NMSSM is a possible solution to the so-called $\mu$ problem, the fact, that the supersymmetric parameter $\mu$ in the superpotential is dimensionful and should in principle be of the order of the SUSY breaking scale. A working electroweak symmetry breaking demands this parameter, however, in the ball park of a few hundred $\mathrm{GeV}$. The NMSSM tackles this problem by enlarging the particle spectrum by a single left-chiral superfield $S$, being a singlet under the SM gauge group. Their are two general options to generate a quartic potential term for the scalar part of $S$ : either a $D$ term of an additional $U(1)$ guage symmetry (like e.g. in the PSSSM [9]) or by a $F$ term from a cubic superpotential term (conventional NMSSM). For the conventions, we follow those of the SUSY Les Houches Accord 2 [10] (for a recent review on NMSSM conventions cf. also [8]). This is a quite general approach, but neglecting possible CP, $R$-parity, or flavor violation. The suporpotential of the NMSSM is given by

$$
W_{N M S S M}=W_{M S S M}-\varepsilon_{a b} \lambda S H_{1}^{a} H_{2}^{b}+\frac{1}{3} \kappa S^{3}+\mu^{\prime} S^{2}+\xi_{F} S,
$$

where we neglect an explicit $\mu^{\prime}$ term and the Fayet-Iliopoulos term for the singlet superfield. The most general soft-breaking terms for the NMSSM

$V_{\mathrm{soft}}=V_{2, M S S M}+V_{3, M S S M}+m_{\mathrm{S}}^{2}|S|^{2}+\left(-\varepsilon_{a b} \lambda A_{\lambda} S H_{1}^{a} H_{2}^{b}+\frac{1}{3} \kappa A_{\kappa} S^{3}+m_{S}^{\prime 2} S^{2}+\xi_{S} S+\right.$ h.c. $)$,

using the conventions from [10].

The field content of the NMSSM is almost the same as for the MSSM, except for an additional scalar and pseudoscalar Higgs boson, denoted by $H_{3}^{0}$ and $A_{2}^{0}$, as well as a fifth neutralino, $\tilde{\chi}_{5}^{0}$ coming from the additional singlino component.

\section{IMPLEMENTATION AND VALIDATION}

As mentioned in the section about the model before, we are sticking to the SLHA 2 conventions. This fixes the uncertainties in signs and phases. As for the MSSM, the 

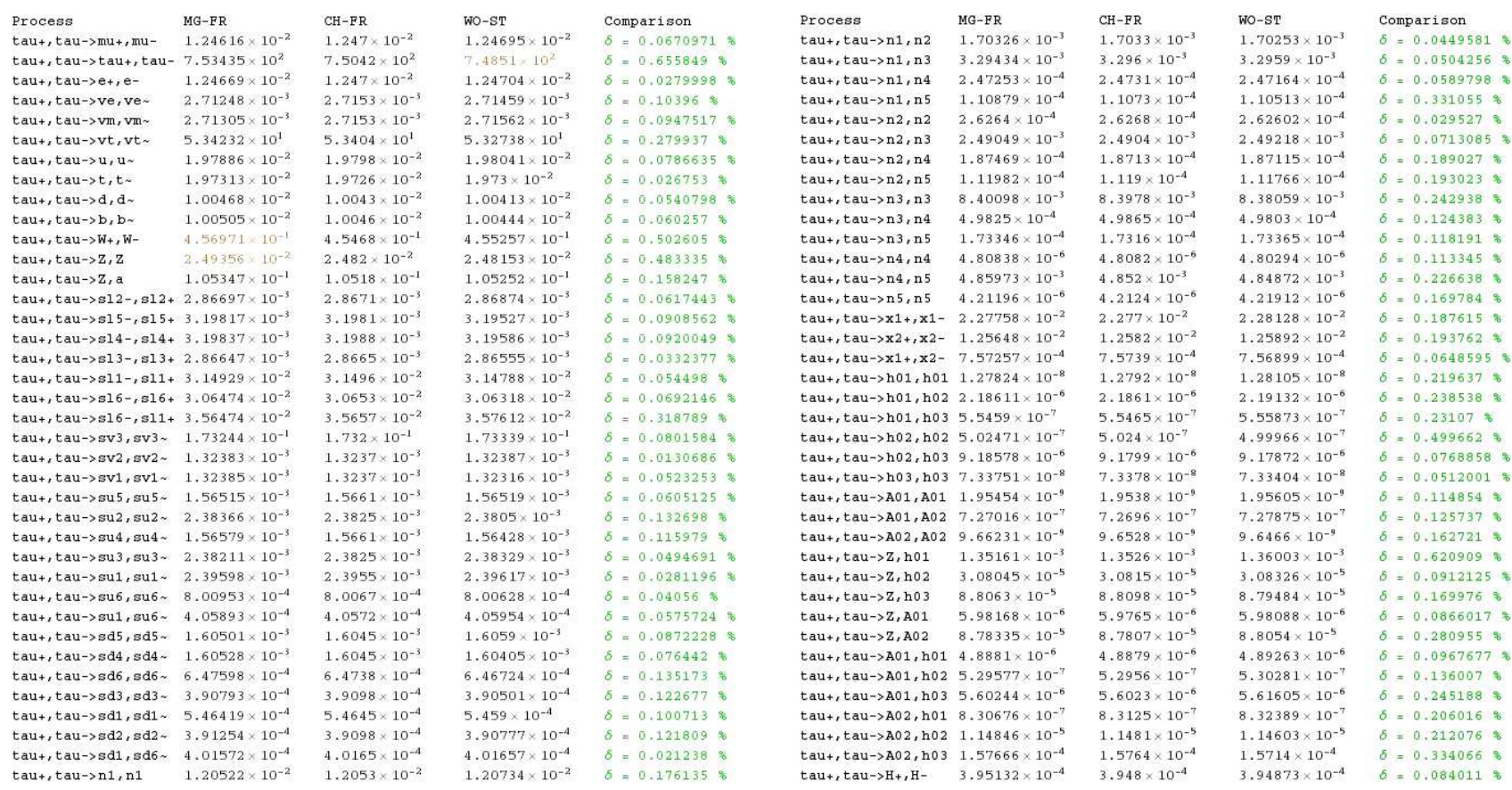

FIGURE 1. Comparison between WHIZARD and the FeynRules implementation within Madgraph. The green color shows agreement, at least up to the Monte Carlo integration error. We used $\sqrt{s}=3 \mathrm{TeV}$ to be above all thresholds.

WHIZARD implementation uses explicitly positive masses for charginos and neutralinos, and puts signs and complex phases instead in the corresponding mixing matrices. In contrast to the MSSM, the NMSSM implementation is more flexible than the MSSM one (although now thanks to Björn Herrmann there is a completely general MSSM implementation in WHIZARD), as it allows not only for a full CKM matrix but also for a left-/right mixing for all generations, but no inter-generational mixing. The generalization to the $\mathrm{CP}$-non-conserving case is easily possible.

The NMSSM is a whole is an incredibly complicated model with order 6,700 couplings (including quartic and Goldstone couplings, most of each are fortunately not of phenomenological importance). It is mandatory to check an implementation as far as possible. For this task, we followed the strategy given in [11]: unitarity checks for $2 \rightarrow 2$ and $2 \rightarrow 3$ scattering amplitudes have been performed, and we tested Ward- and SlavnovTaylor identities for both gauge and supersymmetry. Another check was to reproduce the correct MSSM limit for the NMSSM, namely letting the trilinear singlet Higgs coupling and the cubic singlet coupling approach zero, sending the singlet vev to infinity while keeping the combination of the latter two fixed to $\mu: \lambda \rightarrow 0, \kappa \rightarrow 0,\langle S\rangle \rightarrow \infty,\langle S\rangle \lambda \rightarrow \mu$.

What is by far one of the most stringent tests is the comparison with an independent implementation. For that purpose we are using a FeynRules generated NMSSM model file for MadEvent/Madgraph [12] as well as CalcHEP [13]. Table 11 shows the comparison for $\tau^{+} \tau^{-}$initial state processes as an example. The corresponding SLHA 2 input file is left out here due to reasons of limited space. Our comparison contains far more than 600 processes, showing full agreement between the two implementations. 


\section{CONCLUSIONS}

We presented the complete implementation of the NMSSM implementation into the multi-purpose event generator WHIZARD. A vast sample of consistency checks and comparisons with independent implementations show the correctness of the code. WHIZARD is available as a modern high-level tool for NMSSM signal and background simulations for the LHC era. A detailed version of the comparison and validation of the NMSSM implementations will appear soon [14].

\section{ACKNOWLEDGMENTS}

The authors are supported by the Ministerium für Bildung und Kultur of the state Baden-Württemberg by the program ZO IV and the German Research Society (DFG) under grant no. Re 2850/1-1. JR would like to thank to Aspen Center for Physics for their hospitality. We are also grateful to Ulrich Ellwanger for valuable remarks and discussions, as well as Benjamin Fuks, Neil Christensen, Claude Duhr and Christian Speckner for the FeynRules collaboration.

\section{REFERENCES}

1. W. Kilian, T. Ohl and J. Reuter, arXiv:0708.4233 [hep-ph].

2. T. Ohl, Comput. Phys. Commun. 120, 13 (1999) [arXiv:hep-ph/9806432].

3. M. Moretti, T. Ohl and J. Reuter, arXiv:hep-ph/0102195.

4. J. Reuter, PhD thesis arXiv:hep-th/0212154; T. Ohl and J. Reuter, Eur. Phys. J. C 30, 525 (2003) [arXiv:hep-th/0212224]; W. Kilian, J. Reuter and T. Robens, Eur. Phys. J. C 48, 389 (2006) [arXiv:hep-ph/0607127]; T. Robens, J. Kalinowski, K. Rolbiecki, W. Kilian and J. Reuter, Acta Phys. Polon. B 39, 1705 (2008) arXiv:0803.4161 [hep-ph]]; J. Kalinowski, W. Kilian, J. Reuter, T. Robens and K. Rolbiecki, JHEP 0810, 090 (2008) [arXiv:0809.3997 [hep-ph]].

5. W. Kilian, D. Rainwater and J. Reuter, Phys. Rev. D 71, 015008 (2005) [arXiv:hep-ph/0411213]; Phys. Rev. D 74, 095003 (2006) [arXiv:hep-ph/0609119]; M. Beyer et al., Eur. Phys. J. C 48, 353 (2006) [arXiv:hep-ph/0604048]; A. Alboteanu, W. Kilian and J. Reuter, JHEP 0811, 010 (2008) [arXiv:0806.4145 [hep-ph]].

6. S. Ovyn, X. Rouby and V. Lemaitre, arXiv:0903.2225 [hep-ph].

7. N. D. Christensen and C. Duhr, arXiv:0806.4194[hep-ph]; N. D. Christensen et al., arXiv:0906.2474 [hep-ph].

8. E. Accomando et al., arXiv:hep-ph/0608079

9. W. Kilian and J. Reuter, Phys. Lett. B 642, 81 (2006) [arXiv:hep-ph/0606277].

10. P. Skands et al., JHEP 0407, 036 (2004) [arXiv:hep-ph/0311123]; B. Allanach et al., Comput. Phys. Commun. 180, 8 (2009) [arXiv:0801.0045 [hep-ph]]; J. A. Aguilar-Saavedra et al., Eur. Phys. J. C 46, 43 (2006) [arXiv:hep-ph/0511344].

11. K. Hagiwara et al., Phys. Rev. D 73, 055005 (2006) [arXiv:hep-ph/0512260].

12. J. Alwall et al., JHEP 0709, 028 (2007) [arXiv:0706.2334[hep-ph]]; F. Maltoni and T. Stelzer, JHEP 0302, 027 (2003) [arXiv:hep-ph/0208156].

13. A. Pukhov, arXiv:hep-ph/0412191.

14. F. Braam, B. Fuks, J. Reuter, in preparation. 